\title{
The SPECT/CT Evaluation of Compartmental Changes after Open Wedge High Tibial Osteotomy
}

\author{
Tae Won Kim, $\mathrm{MD}^{1}$, Byung Kag Kim, $\mathrm{MD}^{1}$, Dong Whan Kim, $\mathrm{MD}^{1}$, Jae Ang Sim, $\mathrm{MD}^{1}$, Beom Koo Lee, $\mathrm{MD}^{2}$, \\ and Yong Seuk Lee, $\mathrm{MD}^{3}$ \\ ${ }^{1}$ Department of Orthopedic Surgery, Gacheon University Gil Medical Center, Incheon; ${ }^{2}$ Department of Orthopedic Surgery, The Armed Forces Capital Hospital, \\ Seongnam; ${ }^{3}$ Department of Orthopedic Surgery, Seoul National University College of Medicine, Seoul, Korea
}

\begin{abstract}
Purpose: The purpose of this study was to evaluate compartmental changes using combined single-photon emission computerized tomography and conventional computerized tomography (SPECT/CT) after open wedge high tibial osteotomy (OWHTO) for providing clinical guidance for proper correction.

Materials and Methods: Analysis was performed using SPECT/CT from around 1 year after surgery on 22 patients who underwent OWHTO. Postoperative mechanical axis was measured and classified into 3 groups: group I (varus), group II $\left(0^{\circ}-3^{\circ}\right.$ valgus), and group III ( $>3^{\circ}$ valgus). Patella location was evaluated using Blackburne-Peel (BP) ratio. On SPECT/CT, the knee joint was divided into medial, lateral, and patellofemoral compartments and the brighter signal was marked as a positive signal.

Results: Increased signal activity in the medial compartment was observed in 12 cases. No correlation was observed between postoperative mechanical axis and medial signal increase. Lateral increased signal activity was observed in 3 cases, and as valgus degree increased, lateral compartment's signal activity increased. Increased signal activity of the patellofemoral joint was observed in 7 cases, and significant correlation was observed between changes in $\mathrm{BP}$ ratio and increased signal activity.

Conclusions: For the treatment of medial osteoarthritis, OWHTO requires overcorrection that does not exceed 3 valgus. In addition, the possibility of a patellofemoral joint problem after OWHTO should be kept in mind.
\end{abstract}

Keywords: Knee, Osteotomy, Open wedge, Single-photon emission computerized tomography

\section{Introduction}

The main principle of osteotomy is to achieve a transfer of loading from diseased, arthritic areas of the joint to areas with relatively intact, healthy cartilage. High tibial osteotomy (HTO) is a surgical treatment option for symptomatic medial compartment osteoarthritis of the knee with varus deformity and without lat-

Received August 17, 2015; Revised (1st) December 7, 2015;

(2nd) January 5, 2016; Accepted February 15, 2016

Correspondence to: Beom Koo Lee, MD

Department of Orthopedic Surgery, The Armed Forces Capital Hospital, 81, Saemaeul-ro 177beon-gil, Bundang-gu, Seongnam 13574, Korea

Tel: +82-31-725-6221, Fax: +82-31-706-0987

E-mail: knee5360@naver.com

This is an Open Access article distributed under the terms of the Creative Commons Attribution Non-Commercial License (http://creativecommons.org/licenses/by-nc/4.0/) which permits unrestricted non-commercial use, distribution, and reproduction in any medium, provided the original work is properly cited. eral compartment osteoarthritis or patellofemoral osteoarthritis ${ }^{1)}$. Undercorrection with a persisting varus alignment usually leads to poor results after HTO and overcorrection to a great valgus alignment may result in medial joint opening and rapid development of lateral osteoarthritis after $\mathrm{HTO}^{2}$.

Combined single-photon emission computerized tomography and conventional computerized tomography (SPECT/CT) is a hybrid imaging modality, which combines three-dimensional (3D) bone scintigraphy 'SPECT' and conventional CT into one imaging procedure ${ }^{3-10)}$.

Hirschmann et al. ${ }^{3)}$ reported that a loading pattern of mechanical varus/valgus alignment showed significant correlation with higher SPECT/CT tracer uptake of a specific compartment and a higher risk of osteoarthritis progression. This study by Hirschmann et al. ${ }^{3)}$ demonstrated the possibility that the signal pattern on SPECT/CT could be used in diagnosis of early osteoarthritis. 
Hence, when the signal strength is measured using SPECT/CT after HTO, the risk of arthritis progression can be determined even with a short follow-up. Therefore, the current study was conducted to analyze the usefulness of the surgery using SPECT/ CT.

The purpose of the current study was to examine the effect of a valgization open wedge high tibial osteotomy (OWHTO) on intra-articular cartilage changes of each compartment using SPECT/CT. Our hypothesis was there would be a correlation between the degree of mechanical axis of HTO and SPECT/CT image which can be used as a clinical guide for proper correction.

\section{Materials and Methods}

\section{Materials}

Among the patients who had undergone OWHTO at our hospital between January 2013 and November 2014, 22 patients (22 cases), in whom 1-year postoperative follow-up was possible, were analyzed using SPECT/CT images taken around 1 year after the surgery (average, 12 months postoperatively; range, 11 to 17 months postoperatively). All 22 patients had symptomatic medial compartment osteoarthritis (Kellgren-Lawrence grade II or III) of the knee with varus deformity and had neither lateral compartment osteoarthritis nor patellofemoral osteoarthritis. Two patients had undergone sequential HTO on both sides, and the analysis was performed using data from the first surgery. In all cases, SPECT/CT imaging was performed around 1 year after surgery (average, 12 months postoperatively; range, 11 to 17 months postoperatively) and after verifying bone fusion. Surgery in all cases was performed by a single, experienced orthopedic surgeon.

There were 3 males and 19 females with a mean age of 57.7 years (range, 45 to 74 years) at the time of surgery. Preoperative lower extremity alignment showed an average of $6.93^{\circ}$ of varus mechanical alignment (Table 1).

Prior to the osteotomy, a diagnostic arthroscopy was performed in all cases, and if medial meniscal root tear was detected, repair or partial meniscectomy was performed, whereas if cartilage lesions were detected on the medial tibiofemoral articular surface, intra-articular procedures such as microfracture were performed for cartilage lesions of Outerbridge grade III or higher.

Regarding the medial Outerbridge grade by preoperative diagnostic arthroscopy, 6 cases were grade II, 4 cases were grade III, and 12 cases were grade IV among the 22 cases.

The correction angle was estimated preoperatively using the ruler method with the Fujisawa point set as the target. During surgery, the angle was checked using a C-arm fluoroscope with application of axial force in consideration of weight bearing situations.

Rehabilitation in the first postoperative year, prior to follow-up SPECT, involved walking with crutches and walking with aids immediately after the surgery, with crutches being removed and full weight bearing allowed from the second month on and use of walking aids permitted until the third month. The same rehabilitation regimen was applied to all patients.

SPECT imaging was performed between January 2014 and November 2015, after providing explanations to all study participants on the necessity of SPECT/CT examinations for postoperative follow-up observations and receiving their consent.

This study was approved by the Institutional Review Board of our hospital before commencing the study.

\section{Radiographic Measurement and SPECT/CT Imaging}

In the current study, the mechanical axis at the time of SPECT/ CT imaging was used as the criterion for group classification (Fig. 1).

Postoperative mechanical axis was classified into 3 groups: group I (varus), group II $\left(0^{\circ}-3^{\circ}\right.$ valgus), and group III ( $>3^{\circ}$ valgus).

Although there are slight differences in the most ideal postoperative correction angle reported by each author ${ }^{11-14)}$, the Fujisawa point currently used by many authors aims for $2^{\circ}-4^{\circ}$ of valgus in the mechanical axis and $8^{\circ}-10^{\circ}$ of valgus in the anatomical axis ${ }^{11}$; and as such, the current study divided the patient groups using a cut-off of $3^{\circ}$ of valgus of the mechanical axis.

Patella location was estimated using the preoperative and postoperative Blackburne-Peel (BP) ratio, and the difference was calculated (Fig. 2).

Arthroscopic cartilage lesions were classified according to

Table 1. Gender, Age, and Mechanical Axis (MA) in Each Group

\begin{tabular}{lcccc}
\hline \multicolumn{1}{c}{ Variable } & Group I $(\mathrm{n}=2)$ & Group II $(\mathrm{n}=13)$ & Group III $(\mathrm{n}=7)$ & Total $(\mathrm{n}=22)$ \\
\hline Gender $(\mathrm{M}: \mathrm{F})$ & $0: 2$ & $1: 12$ & $2: 5$ & $3: 19$ \\
Mean age $(\mathrm{yr})$ & 58.50 & 58.15 & 56.71 & 57.73 \\
Preoperative $\mathrm{MA}\left({ }^{\circ}\right)$ & Varus 5.84 & Varus 7.19 & Varus 6.76 & Varus 6.93 \\
Postoperative $\mathrm{MA}\left({ }^{\circ}\right)$ & Varus 1.57 & Valgus 1.47 & Valgus 3.82 & Valgus 1.94 \\
\hline
\end{tabular}


Outerbridge grades. During diagnostic arthroscopy prior to corrective osteotomy, Outerbridge grade assessments on cartilage lesions were performed only on the medial compartment (medial femoral condyle and medial tibial plateau) (Fig. 3).

Using SPECT/CT, we evaluated the signal in 3 compartments of the knee joint (medial, lateral, and patellofemoral compartments). The signal intensity was compared with the tibiofemoral shaft as a comparison group, which showed no signal in all patients. In this comparison, the brighter signal was marked as a positive signal and the same signal intensity as the comparison
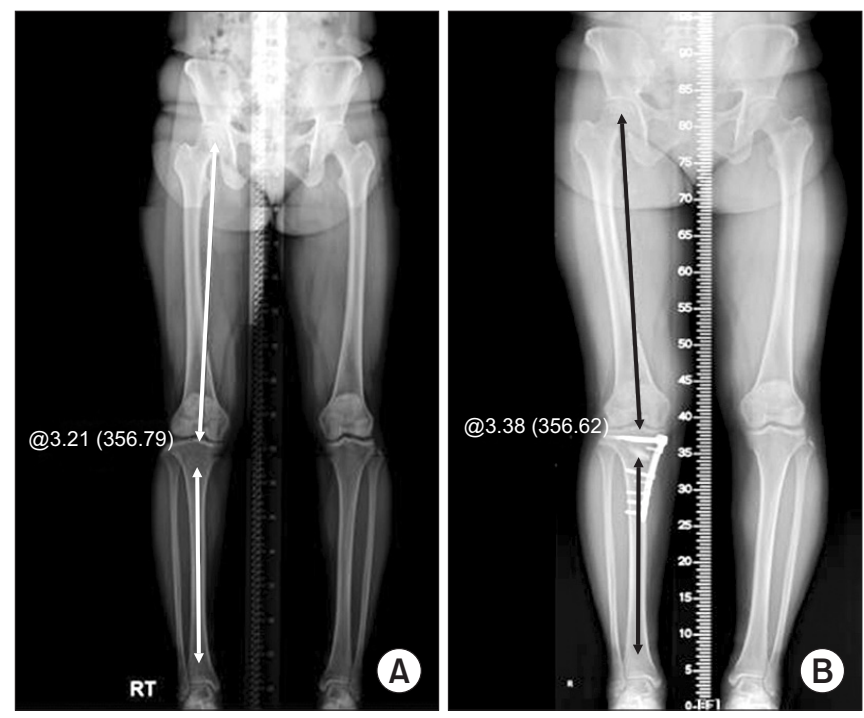

Fig. 1. Preoperative (A) and postoperative (B) measurements and changes in the mechanical axis on full-length lower extremity scanogram (preoperative mechanical axis, $3.21^{\circ}$ varus; postoperative mechanical axis, $3.38^{\circ}$ valgus).
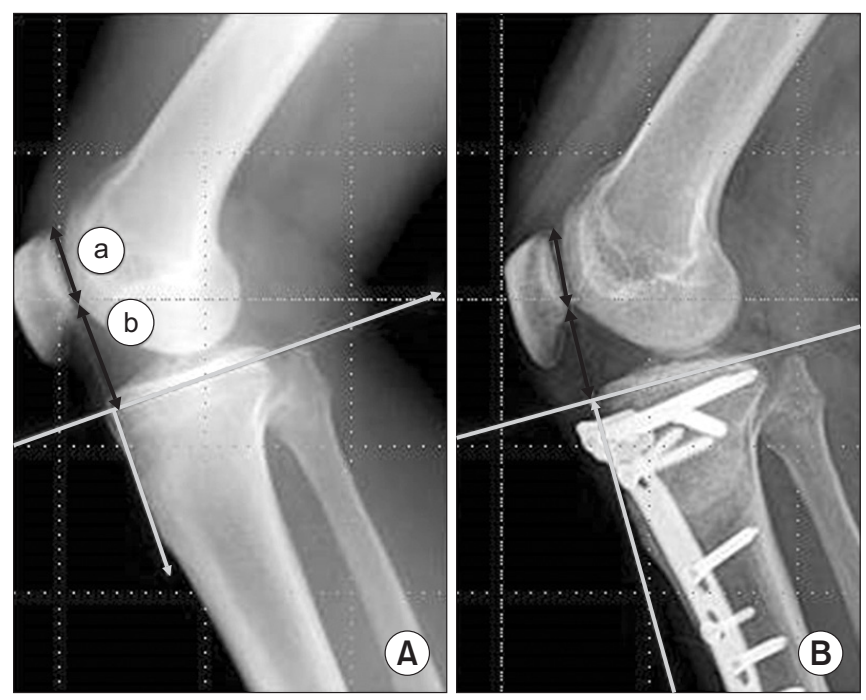

Fig. 2. Measurement of the Blackburne-Peel ratio (b/a) on the preoperative (A) and postoperative (B) lateral radiographs. group was marked as a negative signal (Figs. 4 and 5). The evaluation of signal intensity was performed independently by one orthopedic surgeon and one radiologist who had no association with the surgery performed on the patients, and the results of evaluation by these two observers corresponded completely (Cohen kappa coefficient, 1).

\section{Statistical Analysis}

IBM SPSS ver. 22.0 (IBM Co., Armonk, NY, USA) was used for

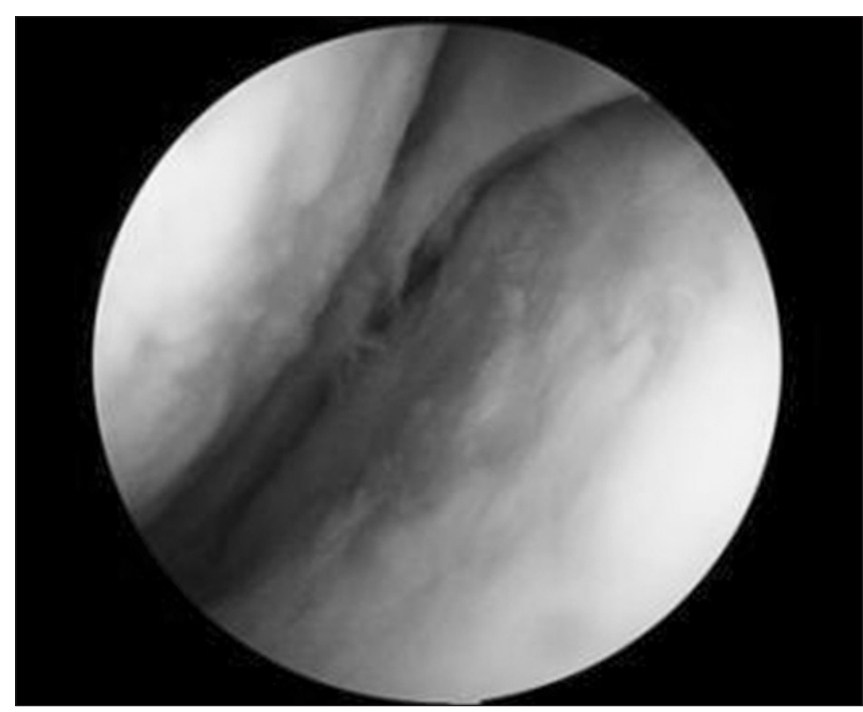

Fig. 3. Medial cartilage lesions were identified by preoperative arthroscopic examination before high tibial osteotomy and classified according to Outerbridge classification.

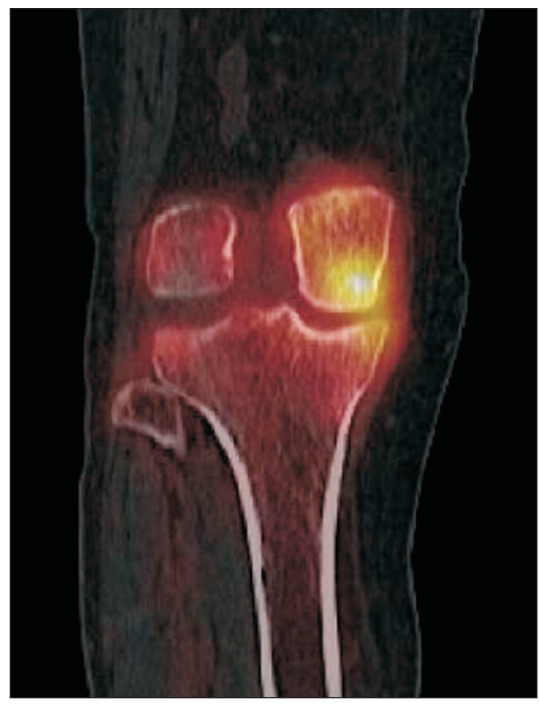

Fig. 4. Positive signal in the medial tibiofemoral compartment on singlephoton emission computerized tomography (CT) and conventional CT (the signal is brighter on the articular side of the medial femoral condyle). 
data analysis; increased signal activity of each compartment was calculated according to groups, BP ratio, and Outerbridge grades using chi-square test, $t$-test. A p-value less than 0.05 was considered statistically significant.

\section{Results}

The preoperative mechanical axis was $1.57^{\circ}$ varus for 2 cases in group I (varus), $1.47^{\circ}$ valgus for 13 cases in group II $\left(0^{\circ}-3^{\circ}\right.$ valgus $)$, and $3.82^{\circ}$ valgus for 7 cases in group III ( $>3^{\circ}$ valgus) (Table 1 ).

\section{Increased Signal Activity in the Medial Compartment by Group and Outerbridge Classification}

Increased signal activity in the medial compartment was observed in 12 cases: $100 \%$ in group I, $46 \%$ in group II, and 57\% in group III (Table 2, Fig. 4). No correlation was observed between postoperative mechanical axis and medial signal increase $(\mathrm{p}=0.301)$. In preoperative Outerbridge classification, medial increased signal activity was shown in $66 \%$ in grade II, $25 \%$ in grade III, and 58\% in grade IV (Table 3 ). No correlation was observed between preoperative Outerbridge classification and

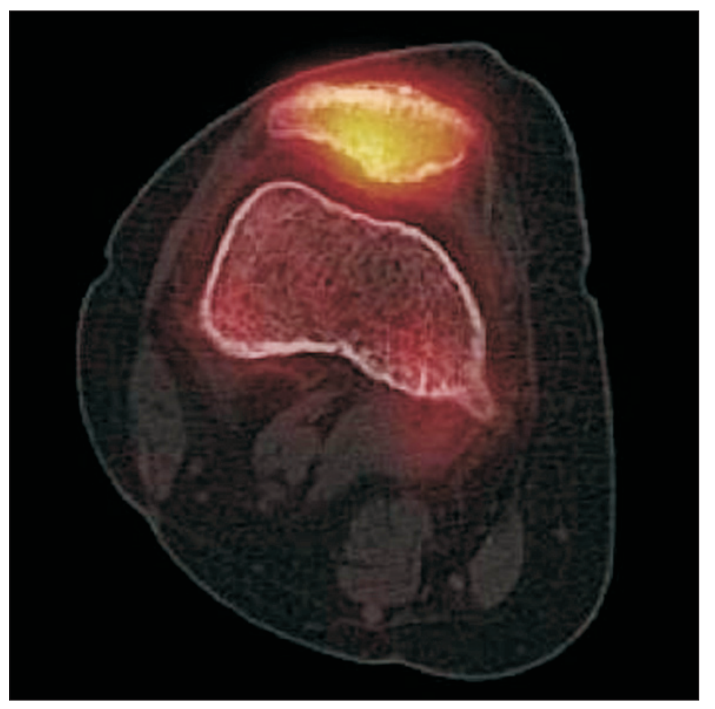

Fig. 5. Positive signal in the patellofemoral compartment on singlephoton emission computerized tomography (CT) and conventional CT (the signal is brighter on the articular side of the patella).

Table 2. Increased Signal Activity in the Medial Compartment by Group

\begin{tabular}{ccccc}
\hline Medial hot uptake & Group I & Group II & Group III & Total \\
\hline- & 0 & 7 & 3 & 10 \\
+ & 2 & 6 & 4 & 12 \\
Total & 2 & 13 & 7 & 22 \\
\hline
\end{tabular}

medial increased signal activity.

\section{Increased Signal Activity in the Lateral Compartment by Group}

Lateral increased signal activity was observed in only 3 cases, and the mean mechanical axis was $3.53^{\circ}$ valgus. All 3 patients belonged to group III and there was significant difference between groups $(\mathrm{p}=0.005)$. When valgus degree increased, lateral compartment signal activity also increased $(\mathrm{p}=0.005)$ (Table 4$)$.

\section{Increased Signal Activity in the Patellofemoral Joint by BP Ratio}

The BP ratio was measured preoperatively and postoperatively. The preoperative value was $0.75 \pm 0.13$ and the postoperative value was $0.67 \pm 0.13$, and the difference was $0.08 \pm 0.4$. Increased signal activity of the patellofemoral joint was observed in 7 cases, and statistically significant correlation was observed between hot uptake in the patellofemoral joint and changes in the BP ratio $(\mathrm{p}=0.017)$ (Table 5, Fig. 5).

Table 3. Increased Signal Activity in the Medial Compartment by Preoperative Outerbridge Classification

\begin{tabular}{ccccc}
\hline Medial hot uptake & Grade II & Grade III & Grade IV & Total \\
\hline- & 2 & 3 & 5 & 10 \\
+ & 4 & 1 & 7 & 12 \\
Total & 6 & 4 & 12 & 22 \\
\hline
\end{tabular}

Table 4. Increased Signal Activity in the Lateral Compartment by Group ${ }^{\text {a) }}$

\begin{tabular}{ccccc}
\hline Lateral hot uptake & Group I & Group II & Group III & Total \\
\hline- & 2 & 13 & 4 & 19 \\
+ & 0 & 0 & 3 & 3 \\
Total & 2 & 13 & 7 & 22 \\
\hline
\end{tabular}

${ }^{a)}$ Linear by linear association, $\mathrm{p}=0.005$.

Table 5. Increased Signal Activity in the Patellofemoral Joint by BP Ratio ${ }^{\text {a) }}$

\begin{tabular}{lccc}
\hline \multicolumn{1}{c}{ Variable } & $\begin{array}{c}\text { Patellofemoral } \\
\text { uptake }-(\mathrm{n}=15)\end{array}$ & $\begin{array}{c}\text { Patellofemoral } \\
\text { uptake }+(\mathrm{n}=7)\end{array}$ & p-value \\
\hline Preoperative BP ratio & $0.69 \pm 0.16$ & $0.82 \pm 0.84$ & 0.019 \\
Postoperative BP ratio & $0.63 \pm 0.16$ & $0.71 \pm 0.07$ & 0.159 \\
Difference & $0.06 \pm 0.04$ & $0.11 \pm 0.02$ & 0.001 \\
\hline
\end{tabular}

BP: Blackburne-Peel.

${ }^{a}$ Linear by linear association, $\mathrm{p}=0.017$. 


\section{Discussion}

The clinical uses of SPECT/CT have increased recently because biologic activities observed on SPECT have been known to strictly correlate with the anatomical region observed on $\mathrm{CT}^{3-10)}$. Hirschmann et al. ${ }^{3)}$ reported that a loading pattern by mechanical varus/valgus alignment showed significant correlation with higher SPECT/CT tracer uptake of specific compartment and higher risk of osteoarthritis progression. In the report, SPECT/ CT was used as an imaging modality for follow-up of patients after realignment treatment such as HTO.

In addition, Hirschmann et al. ${ }^{4)}$ reported that combining the three-dimensional (3D) mechanical information on tunnel placement and attachment areas and the 3D metabolic data using SPECT/CT would be helpful in evaluation of patients with pain after anterior cruciate ligament reconstruction and causes of graft failure. Konala et al. ${ }^{7)}$ reported that SPECT/CT may hold great clinical value for symptomatic patients who have previously undergone treatment for osteochondral lesions. Hirschmann et al. ${ }^{8-10)}$ reported on the value of SPECT/CT for evaluation of patients with unexplained pain after total knee arthroplasty or patellofemoral disorders.

In addition, plane SPECT reflects serum markers of bone and cartilage metabolism such as cartilage oligomeric matrix protein and bone sialoprotein. As subchondral bone changes precede joint space narrowing, SPECT can detect osteoarthritis earlier than radiography ${ }^{15,16}$. The SPECT/CT systems can provide clear anatomical detail through high resolution CT imaging.

Despite increased interest in valgization HTO, there is little information on the intra-articular effect of osteotomy. The SPECT/ CT reflects the mechanical loading of different compartments in the knee. We studied open wedge proximal osteotomy patients with SPECT/CT to investigate its use as a clinical guide for proper correction. In all cases of the varus group, the medial compartment showed hot uptake, and hot uptake in the lateral compartment was seen when the mechanical axis exceeded $3^{\circ}$ of valgus. In the group that showed an increase in signal in the patellofemoral joint on the SPECT/CT at 1 year after surgery, statistically significant differences were observed in $\mathrm{BP}$ ratios before and after surgery.

In our study, lateral increased signal activity was observed in 3 cases, and their mean mechanical axis was $3.53^{\circ}$ valgus. All of these patients belonged to group III, and statistically significant difference was observed between groups $(\mathrm{p}=0.005)$. Therefore, we thought that overcorrection was necessary; however, overcorrection over $3^{\circ}$ of valgus of the mechanical axis would be harmful to the lateral compartment regarding the progression of osteoarthritis.

Fujisawa et al. ${ }^{11)}$ recommended using the weight bearing line through a point at $62 \%$ of the tibial width through the lateral compartment. Coventry ${ }^{12)}$ stated that the survival rate could be $94 \%$ when the femorotibial angle was $8^{\circ}-10^{\circ}$ valgus and $64 \%$ when it was less than $8^{\circ}$ valgus. Rudan and Simurda ${ }^{13)}$ recommended that the ideal femorotibial angle after osteotomy should be $6^{\circ}-14^{\circ}$. Insall et al. ${ }^{14)}$ suggested the ideal femorotibial angle as $5^{\circ}-14^{\circ}$.

Mucha et al. ${ }^{17)}$ reported on the decrease of significant signal lesion between the preoperative and postoperative period following HTO. Our study found medial increased signal activity in 12 out of 22 cases. Especially, all patients of group I (varus) showed positive signal intensity in the medial compartment. Thus, we think that surgeons need to overcorrect HTO for the treatment of medial osteoarthritis.

If preoperative osteoarthritis is more severe, we would predict that the medial signal activity would continue after HTO, but even in Outerbridge grade IV, medial hot uptake was only 58\%, and there was no statistically significant difference between the preoperative Outerbridge grade and the signal intensity of SPECT/CT (Table 3). Generally, HTO is not competitive compared to TKA for advanced osteoarthritic patients, but we think that HTO could be considered as an effective operation even in minor cases of advanced osteoarthritis. For example, Schuster et al. ${ }^{18)}$ recently reported that achievement of successful outcomes was possible via treatment of severe medial osteoarthritis and varus malalignment with medial OWHTO combined with a chondral resurfacing procedure (abrasion/microfracture) in cases of Kellgren-Lawrence grade 3 and 4 osteoarthritis with fullthickness cartilage defects and varus malalignment.

Increased signal activity of the patellofemoral joint was observed in 7 cases (32\%). Lee et al. ${ }^{19)}$ reported that patella tilt did not change but patella baja appeared after HTO. In addition, Chae et al. ${ }^{20)}$ reported that the mean BP ratio declined from $0.71 \pm$ 0.12 to $0.61 \pm 0.13(\mathrm{p}<0.001)$ after OWHTO.

In this study, the value of $\mathrm{BP}$ ratio was $0.75 \pm 0.13$ preoperatively and $0.67 \pm 0.13$ postoperatively, and the difference was $0.08 \pm 0.4$ $(\mathrm{p}<0.001)$. In addition, there was statistically significant difference between the BP ratio and the signal activity of the patellofemoral joint $(\mathrm{p}=0.017)$. Therefore, surgeons should also be aware that a patellofemoral joint problem could occur after HTO operation, and selection of patients with operational indication is important.

The limitations of this study were as follows. First, we did not 
analyze the uptake signal on SPECT/CT quantitatively but only examined the appearance of uptake signal. Although SPECT/CT has recently proven useful as a diagnostic tool in the orthopedic field, its use has a number of limitations. Its clinical adoption has been limited due to shortcomings of the available analytical tools. Specifically, SPECT analyses are mainly qualitative due to variation in the overall metabolic uptake among patients. Furthermore, most analyses are performed in two-dimensional, despite the wide availability of 3D data. Consequently, quantitative comparison of the position, size, and intensity of SPECT uptake regions among patients is difficult; therefore, it is difficult to draw meaningful clinical conclusions. Second, due to the small number of patients, it is difficult to grant statistical significance to the findings of the study. A power analysis for calculation of sample size based on this study involving an adequate number of cases would be necessary. Third, preoperative SPECT/CT image scanning was not performed in this study; therefore, we could not perform preoperative and postoperative comparison. In particular, lateral and patellofemoral compartments were not properly analyzed by preoperative SPECT/CT before HTO. As a result, the analysis based only on the 1-year postoperative SPECT/CT could not be conclusive enough to determine clinical correlation without an analysis of arthroscopic changes on the lateral and patellofemoral compartments. In addition, a long-term followup is needed to confirm that the results obtained with SPECT/ CT correspond with those obtained using other radiologic methods. Finally, in this study, we did not consider patient-associated variables such as the physical activity level, quadriceps muscle strength, body mass index, rehabilitation for the 1-year postoperative period, and the extent of postoperative activity.

\section{Conclusions}

The SPECT/CT is useful in the evaluation of compartment changes in the early postoperative period after OWHTO. For the treatment of medial osteoarthritis, overcorrection is necessary during OWHTO; however, care should be taken not to overcorrect more than $3^{\circ}$ valgus at the same time. In addition, the surgeon should keep in mind that a patellofemoral joint problem could occur after OWHTO.

\section{Conflict of Interest}

No potential conflict of interest relevant to this article was reported.

\section{References}

1. Lee DC, Byun SJ. High tibial osteotomy. Knee Surg Relat Res. 2012;24:61-9.

2. Hui C, Salmon LJ, Kok A, Williams HA, Hockers N, van der Tempel WM, Chana R, Pinczewski LA. Long-term survival of high tibial osteotomy for medial compartment osteoarthritis of the knee. Am J Sports Med. 2011;39:64-70.

3. Hirschmann MT, Schon S, Afifi FK, Amsler F, Rasch H, Friederich NF, Arnold MP. Assessment of loading history of compartments in the knee using bone SPECT/CT: a study combining alignment and 99mTc-HDP tracer uptake/distribution patterns. J Orthop Res. 2013;31:268-74.

4. Hirschmann MT, Mathis D, Afifi FK, Rasch H, Henckel J, Amsler F, Wagner CR, Friederich NF, Arnold MP. Single photon emission computerized tomography and conventional computerized tomography (SPECT/CT) for evaluation of patients after anterior cruciate ligament reconstruction: a novel standardized algorithm combining mechanical and metabolic information. Knee Surg Sports Traumatol Arthrosc. 2013;21:965-74.

5. Hirschmann MT, Wagner CR, Rasch H, Henckel J. Standardized volumetric 3D-analysis of SPECT/CT imaging in orthopaedics: overcoming the limitations of qualitative $2 \mathrm{D}$ analysis. BMC Med Imaging. 2012;12:5.

6. Bybel B, Brunken RC, DiFilippo FP, Neumann DR, Wu G, Cerqueira MD. SPECT/CT imaging: clinical utility of an emerging technology. Radiographics. 2008;28:1097-113.

7. Konala P, Iranpour F, Kerner A, Rasch H, Friederich NF, Hirschmann MT. Clinical benefit of SPECT/CT for followup of surgical treatment of osteochondritis dissecans. Ann Nucl Med. 2010;24:621-4.

8. Hirschmann MT, Davda K, Iranpour F, Rasch H, Friederich NF. Combined single photon emission computerised tomography and conventional computerised tomography (SPECT/CT) in patellofemoral disorders: a clinical review. Int Orthop. 2011;35:675-80.

9. Hirschmann MT, Iranpour F, Konala P, Kerner A, Rasch H, Cobb JP, Friederich NF. A novel standardized algorithm for evaluating patients with painful total knee arthroplasty using combined single photon emission tomography and conventional computerized tomography. Knee Surg Sports Traumatol Arthrosc. 2010;18:939-44.

10. Hirschmann MT, Iranpour F, Davda K, Rasch H, Hugli R, Friederich NF. Combined single-photon emission computerized tomography and conventional computerized tomog- 
raphy (SPECT/CT): clinical value for the knee surgeons? Knee Surg Sports Traumatol Arthrosc. 2010;18:341-5.

11. Fujisawa Y, Masuhara K, Shiomi S. The effect of high tibial osteotomy on osteoarthritis of the knee: an arthroscopic study of 54 knee joints. Orthop Clin North Am. 1979;10:585608.

12. Coventry MB. Upper tibial osteotomy for osteoarthritis. J Bone Joint Surg Am. 1985;67:1136-40.

13. Rudan JF, Simurda MA. High tibial osteotomy: a prospective clinical and roentgenographic review. Clin Orthop Relat Res. 1990;(255):251-6.

14. Insall JN, Joseph DM, Msika C. High tibial osteotomy for varus gonarthrosis: a long-term follow-up study. J Bone Joint Surg Am. 1984;66:1040-8.

15. Naslund JE, Odenbring S, Naslund UB, Lundeberg T. Diffusely increased bone scintigraphic uptake in patellofemoral pain syndrome. Br J Sports Med. 2005;39:162-5.

16. Lorberboym M, Minski I, Macadziob S, Nikolov G, Schachter P. Incremental diagnostic value of preoperative 99mTc-MIBI SPECT in patients with a parathyroid adeno- ma. J Nucl Med. 2003;44:904-8.

17. Mucha A, Dordevic M, Testa EA, Rasch H, Hirschmann MT. Assessment of the loading history of patients after high tibial osteotomy using SPECT/CT: a new diagnostic tool and algorithm. J Orthop Surg Res. 2013;8:46.

18. Schuster P, Schulz M, Mayer P, Schlumberger M, Immendoerfer M, Richter J. Open-wedge high tibial osteotomy and combined abrasion/microfracture in severe medial osteoarthritis and varus malalignment: 5-year results and arthroscopic findings after 2 years. Arthroscopy. 2015;31: 1279-88.

19. Lee YS, Lee SB, Oh WS, Kwon YE, Lee BK. Changes in patellofemoral alignment do not cause clinical impact after openwedge high tibial osteotomy. Knee Surg Sports Traumatol Arthrosc. 2016;24:129-33.

20. Chae DJ, Shetty GM, Lee DB, Choi HW, Han SB, Nha KW. Tibial slope and patellar height after opening wedge high tibia osteotomy using autologous tricortical iliac bone graft. Knee. 2008;15:128-33. 